\title{
Performance of rapid-grown KDP crystals with continuous filtration
}

\author{
Guohang $\mathrm{Hu}^{1}$, Yueliang Wang ${ }^{1,2}$, Junxiu Chang ${ }^{1,2}$, Xiaoyi Xie ${ }^{1,2}$, Yuanan Zhao ${ }^{1}$, Hongji $\mathrm{Qi}^{1}$, and \\ Jianda Shao ${ }^{1}$ \\ ${ }^{1}$ Key Laboratory of Materials for High Power Laser, Shanghai Institute of Optics and Fine Mechanics, Chinese Academy of Sciences, \\ Shanghai 201800, China \\ ${ }^{2}$ University of Chinese Academy of Sciences, Beijing 100049, China \\ (Received 2 January 2015; revised 12 February 2015; accepted 4 March 2015)
}

\begin{abstract}
Rapid growth processing of KDP crystals was improved by employing continuous filtration to eliminate bulk defects. The performances of the KDP crystals, including scattering defects, laser damage resistance and transmittance, were measured and analyzed. Compared with rapid-grown KDP without continuous filtration, the transmittance in the nearinfrared was increased by at least $2 \%$, almost all of 'micron size' defects were eliminated and 'sub-micron size' defects were decreased by approximately $90 \%$. Laser damage testing revealed that the laser-induced damage thresholds (LIDTs), as well as the consistency of the LIDTs from sample to sample, were improved greatly. Moreover, it identified that 'micron size' defects were the precursors which initiated laser damage at relative lower laser fluence $\left(4-6 \mathrm{~J} \mathrm{~cm}{ }^{-2}\right)$, and there was a lower correlation between smaller size scattering defects and laser damage initiation. The improved consistency in the LIDTs, attributed to elimination of 'micron size' defects, and LIDT enhancement originated from the decreased absorption of the KDP crystals.
\end{abstract}

Keywords: continuous filtration; defect; KDP; laser damage; rapid growth

\section{Introduction}

$\mathrm{KH}_{2} \mathrm{PO}_{4}$ (KDP) crystals are important nonlinear optical materials in many applications, such as frequency converters and Pockel cells for high-power large-aperture laser systems $^{[1]}$. In the $1990 \mathrm{~s}$, the rapid growth technique was developed to grow large-scale $(40-50 \mathrm{~cm}) \mathrm{KDP}$ boules at a rate of $10-20 \mathrm{~mm} /$ day $^{[2,3]}$, which was one of the seven wonders during NIF laser construction ${ }^{[4]}$. Due to a growth rate an order of magnitude faster than conventional growth, a greater number of bulk defects were formed, degrading the performance of the crystals by causing pinpoint damage and optical losses, which adversely affected the quality of the downstream beam ${ }^{[1,5]}$. It was found that typical damage pinpoints were caused by defects, which efficiently absorbed laser energy, leading to a temperature rise and subsequent

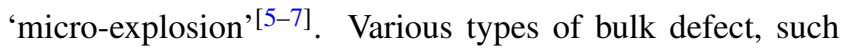
as metallic impurities ${ }^{[8-10]}$, electronic defects ${ }^{[11-13]}$, organic materials, bacteria ${ }^{[14,15]}$, dislocation ${ }^{[16]}$ and insoluble impurities, have been reported. In 1999, the continuous filtration

Correspondence to: Y. Zhao, No. 390, Qinghe Road, Jiading, Shanghai, China. Email: yazhao@siom.ac.cn technique was employed to improve the purity of the growth solution, and high-quality large-scale KDP crystal boules grown at a rate of $12-15 \mathrm{~mm} /$ day were demonstrated ${ }^{[17]}$. Although numerous studies have attempted to show the advantage of continuous filtration during rapid growth processing, no direct evidence has elucidated the reasons for the performance improvement. In the current study, the fundamental reason for the performance improvement was revealed via a comparison of the bulk defect properties, laser damage process and transmittance variation.

\section{Experimental details}

\subsection{Growth of KDP crystals}

KDP crystals were grown rapidly from an over-saturated aqueous solution of high-purity KDP powder in a $45 \mathrm{~L}$ growth vessel equipped with a continuous filtration unit, as shown in Figure 1. Both the growth vessel and the continuous filtration unit were placed in a thermostatic water bath. Two kinds of filter (the first-level filter was of the order of $0.1 \mu \mathrm{m}$, the second-level filter was of the order of $0.01 \mu \mathrm{m}$ ) 


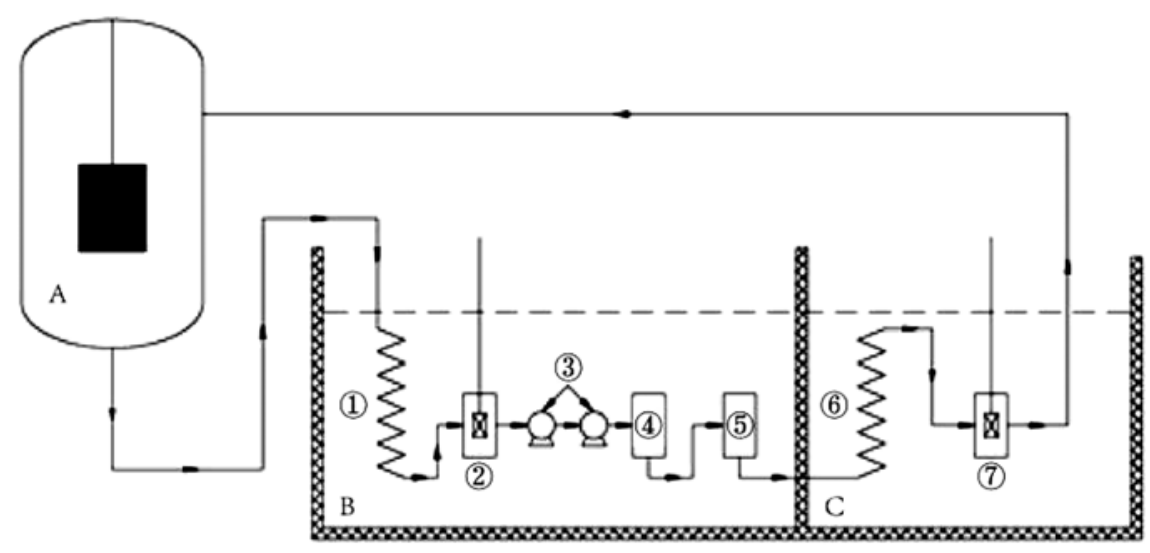
A growth tank
(1) heating section
(4) first-level filter (on the order of $0.1 \mu \mathrm{m}$ )
$B$ heating tank
(2)\&(7) temperature sensor
(5) the second filter (on the order of $0.01 \mu \mathrm{m}$ )
C cooling tank
(3) pumps
(6) heating section

Figure 1. Schematic system for rapid growth of KDP boules using continuous filtration.

were connected inside the continuous filtration unit. A well-polished Z-cut crystal of size $8 \mathrm{~mm} \times 8 \mathrm{~mm} \times 4 \mathrm{~mm}$ was fixed in the center of the growth plate to initiate the crystallization. The growth rate was controlled to $10 \mathrm{~mm} /$ day by lowering the temperature from 318 to $305 \mathrm{~K}$. The crystal was rotated in the 'forward-stop-backward' mode at a speed of 70 r.p.m. The temperature was controlled by a programmable Shimada controller (Model FP21) with an accuracy of $0.02 \mathrm{~K}$. The KDP plates, labeled as CF-1 to CF-6, were cut in a Type I second harmonic generation (SHG) orientation from the same boule prepared by rapid growth with continuous filtration. A further six Type I KDP plates, labeled NCF-1 to NCF-6, prepared by rapid growth without continuous filtration, were chosen as a comparison. All KDP plates were cut to a size of $50 \mathrm{~mm} \times 50 \mathrm{~mm} \times 10 \mathrm{~mm}$. They were annealed at $165^{\circ} \mathrm{C}$ and polished by diamond turning before taking measurements.

\subsection{Measurement of transmittance spectra}

The transmittance spectra of the samples were measured with a Lambda $1050 \mathrm{UV} / \mathrm{Vis} / \mathrm{NIR}$ spectrophotometer in the wavelength range 300-1100 $\mathrm{nm}$ and are shown in Figure 2.

It was found that the transmittance of the CF series samples was slightly (approximately $0.2 \%-1 \%$ ) higher than the NCF series samples in the wavelength range $300-850 \mathrm{~nm}$, and at least $2 \%$ higher than the NCF series samples in the wavelength range $850-1100 \mathrm{~nm}$.

\subsection{Detection of bulk defects}

Defects embedded in the crystals were detected by scattered light microscopy using high-intensity dark-field illumination (Figure 3). A CW $532 \mathrm{~nm}$ laser with a Gaussian spatial

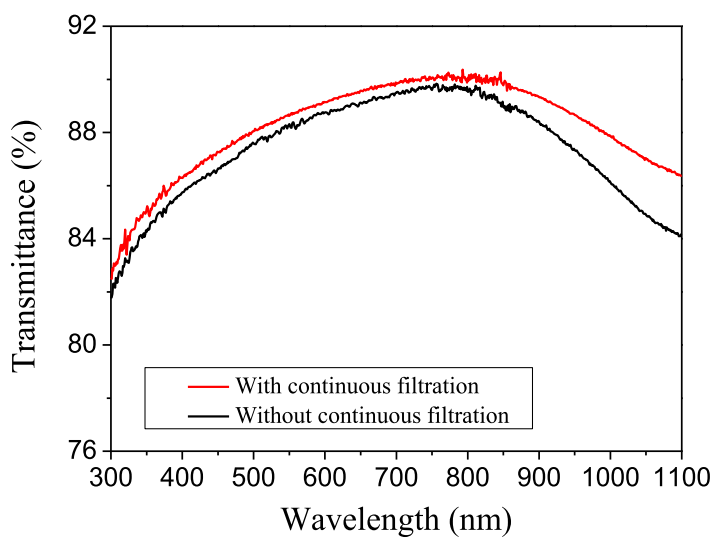

Figure 2. Transmittance spectra of KDP crystals.

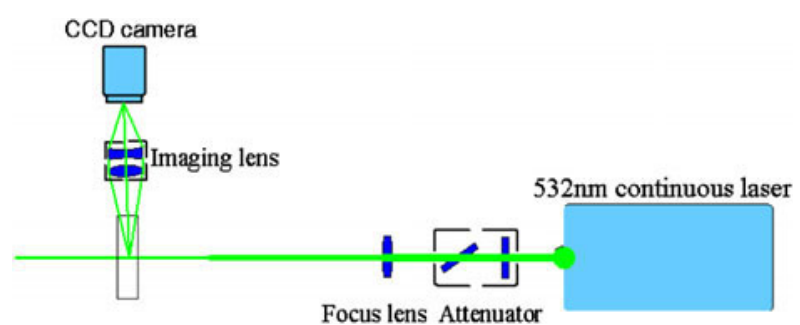

Figure 3. Schematic of the detection system for bulk defects in KDP crystals.

profile was focused and illuminated the bulk site at a power of $1.3 \mathrm{~W}$. The focal length was $5000 \mathrm{~mm}$, and the effective area of the spot on the sample was $0.8 \mathrm{~mm}^{[2]}$. The image of the illuminated region was captured, orthogonal to the direction of laser propagation, through the side of the sample.

Typical scattering defects of the KDP plates are shown in Figure 4. Because the scattering light magnified the 
(a)

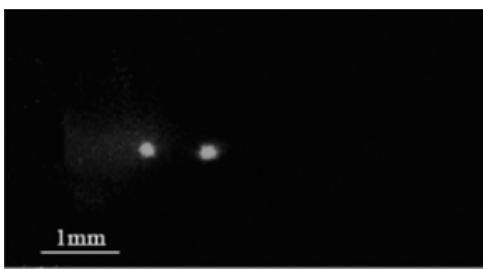

(b)

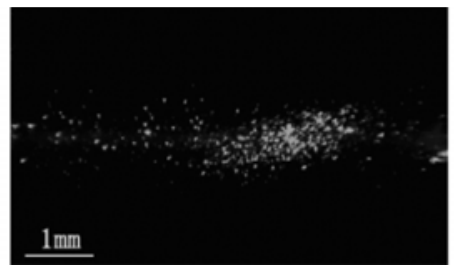

(c)

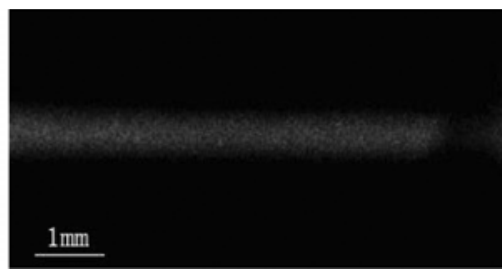

Figure 4. Typical scattering defects (a) 'micron size', (b) 'sub-micron size', (c) 'nano-scale cluster'.

defects and it was difficult to determine their actual size, the defects were categorized by their estimated size in this study. There were three kinds of scattering defect: sparsely distributed 'micron size' defects, high-density 'sub-micron size' defects and 'nano-scale' defect clusters. Statistical analysis of laser damage tests on $70-\mu \mathrm{m}$ defects showed that all of the 'micron size' defects could generate laser damage at fluences higher than $4-6 \mathrm{~J} \mathrm{~cm}^{-2}$. 'Micron size' scattering defects were the most dangerous laser damage initiator. A fraction of 'sub-micron size' defects could lead to laser damage, with the probability of damage initiated being approximately 5\%-40\%. An increased local density of nano-scale defect clusters could also act as an initiator of laser damage.

The size of 'micron size' scattering defects was determined by microscope. The sample was wet-etched to bring the defects to the surface or near to the surface, where the size of the defects could be determined using a Nomarski microscope with $200 \times$ magnification. Figure 5 shows the morphology of a typical 'micron size' scattering defect. The color of 'micron size' defects was different from the surrounding KDP crystal. As is well known, the contrast in bright-field imaging depends on differences in light absorption and the refractive index. It is clear that the absorption or the refractive index of 'micron size' defects was different from the DKDP material. The sizes of the defects shown in Figures 4(b) and 4(c) were not determined in this way, and merely estimated from the scattered intensity, as it was very difficult to distinguish these defects after wet etching.

Due to the variations in the distribution of scattering defects, the density of defects was analyzed statistically by sampling 30 dark images randomly for each sample, as summarized in Figure 6. The density of bulk defects is the number of scattering sites ('micron size' and 'submicron size' defects) divided by the illuminated volume of the KDP crystal. For 'nano-scale clusters', it is more difficult to calculate the density than for the 'micron size' and 'sub-micron size' defects. The probability of capturing these defects from 30 dark images was calculated, and the difference of the brightness was not considered.

It was found that the 'micron size' defects were sparsely distributed, and the density of this kind of defect was approximately $0.008 \mathrm{~mm}^{-3}$ for the NCF series samples. However, they were eliminated after employing continuous

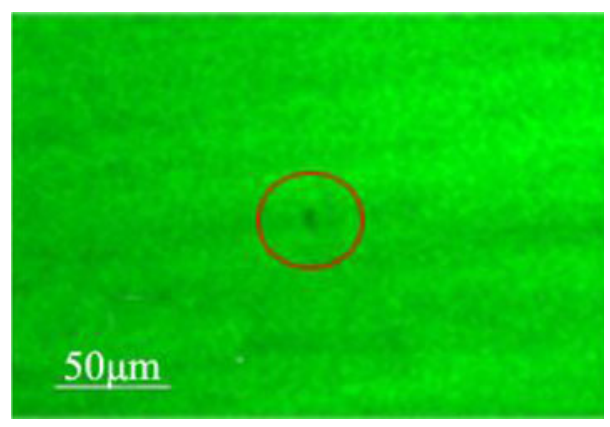

Figure 5. Image of a 'micron size' defect captured by the optical microscope.

filtration. Furthermore, the density of 'sub-micron size' defects in the $\mathrm{CF}$ samples was approximately one or two orders of magnitude lower than that of NCF samples. For the 'nano-scale clusters', although the probability of viewing this kind of defect in CF series samples was slightly lower than in NCF series samples, there was no obvious difference between the two series.

\subsection{Laser damage testing}

The damage test experiments were performed using a pulsed Nd:YAG laser operating at $30 \mathrm{~Hz}$ with an approximate output energy of $1.5 \mathrm{~J}$ at $1064 \mathrm{~nm}$. The pulse duration was approximately $12 \mathrm{~ns}$. The laser fluence on the target plane was adjusted by means of an energy attenuator, consisting of a half-wave plate and a polarizer. The laser beam was focused by the focusing lens onto the target plane. The focal length was $5000 \mathrm{~mm}$, and the effective spot size on the sample was $0.6 \mathrm{~mm}^{2}$, as measured by a laser beam analyzer. The onset of laser damage was detected by an online imaging system with a resolution of the order of microns. A ramp (R-on-1) test was employed to analyze the laser damage resistance of the KDP witnesses, and at least 50 sites on each witness were sampled by laser pulses. The testing results are shown in Figure 7.

It was clear that the laser-induced damage probability shifted to higher fluences after employing continuous filtration. For the NCF series samples, some samples (NCF-3, NCF-4 and NCF-6) exhibited very low laser-induced damage thresholds (LIDTs), attributed to individual damage initiated 
(a)

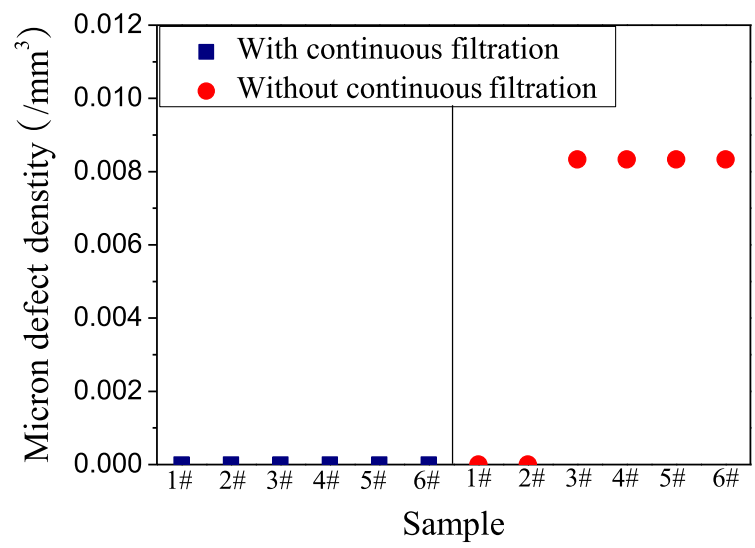

(b)

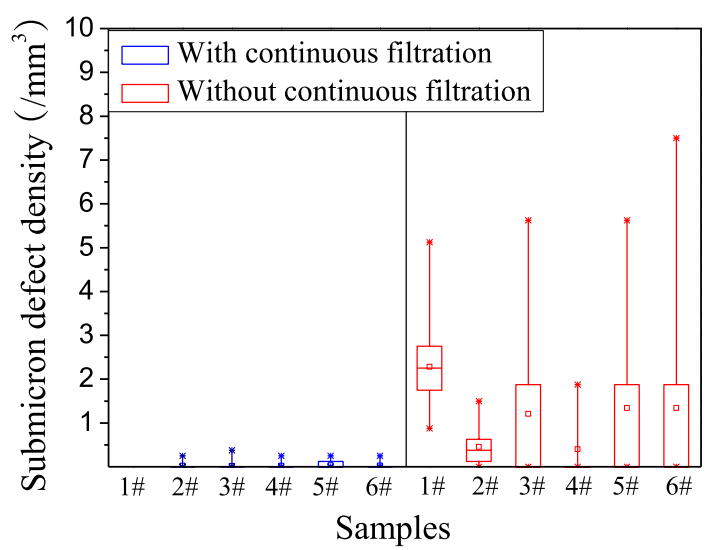

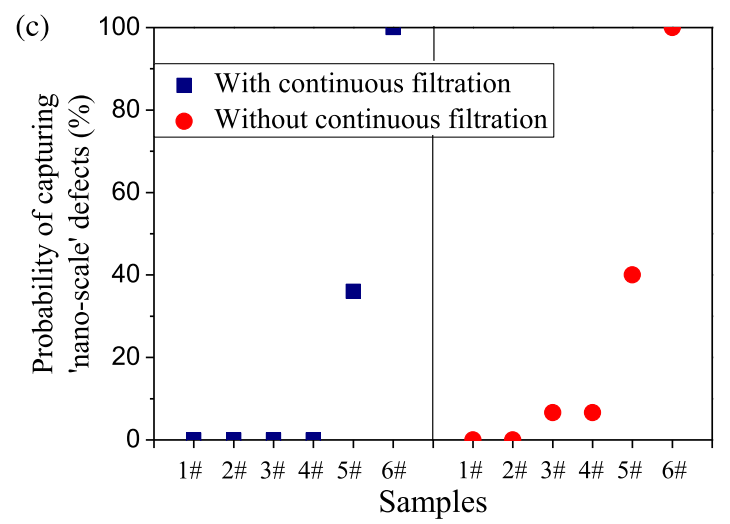

Figure 6. Comparison of (a) 'micron size' scattering defects, (b) 'sub-micron size' scattering defects and (c) 'nano-scale cluster' between the samples grown with and without continuous filtration.

at a very low laser fluence. The LIDTs of the NCF series samples ranged from 2.3 to $22.4 \mathrm{~J} \mathrm{~cm}^{-2}$. For the $\mathrm{CF}$ series samples, the LIDTs range from 25.4 to $36.6 \mathrm{~J} \mathrm{~cm}^{-2}$, and the consistency of the LIDTs was also improved.

\section{Discussion}

By using two-level filters in the continuous filtration system, the new growth method ruled out the probability of inclusions with sizes larger than the filter hole. The statistical data for the scattering defects showed that there were no 'micron size' defects after filtration and 'sub-micron size' defects decreased by $90 \%$. This means that most of the defects with sizes larger than tens of microns were eliminated. However, there was no obvious change in 'nano-scale' defect clusters before and after employing continuous filtration, given its smaller size than the filter holes.

A decreased level of scattering defects reduced scattering losses, which was helpful in improving the transmittance of the KDP crystals. However, there was more noticeable improvement in transmittance for infrared wavelengths than in the visible and UV bands after employing continuous

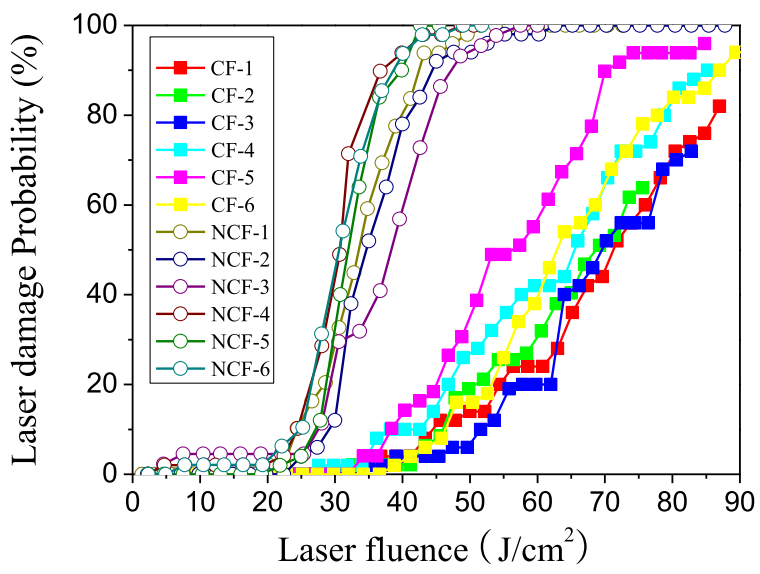

Figure 7. R-on-1 laser damage probability curves for the KDP samples.

filtration; this cannot be attributed to decreased scattering, because one would expect a higher scattering intensity at short wavelengths according to the scattering law. The higher transmittance of the CF series samples in the infrared band mainly comes from the decreased absorption of the KDP crystals. 


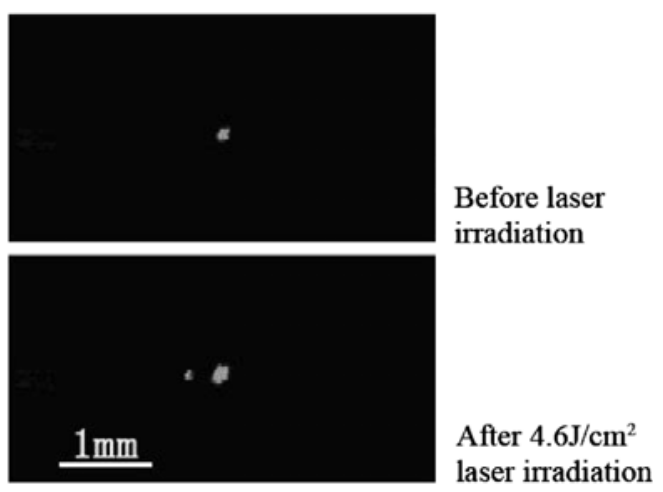

Figure 8. Laser damage initiated at 'micron size' defects in NCF series samples.

For KDP crystals, the prevailing consensus for laser damage initiation has been that the damage is dominated by precursor defects that efficiently absorb the laser energy, and that several kinds of defect may be responsible for damage initiation, such as impurities ${ }^{[18]}$, interstitial atoms or vacancies ${ }^{[19]}$. The online monitoring technique, combining the light-scattering technique and LIDT testing, demonstrated that scattering defects were one of the damage precursors. Figure 8 showed that pinpoint damage initiated from the 'micron size' scattering defects.

The typical laser fluence for damage initiation from 'micron size' defects was approximately $4-6 \mathrm{~J} \mathrm{~cm}^{-2}$, and could be defined as a low-fluence damage precursor. This was the reason for the low LIDT for three of the samples (NCF-3, NCF-4 and NCF-6) and the wide range of LIDTs of NCF series samples. Statistical analysis of the defect density showed that this kind of low-fluence damage precursor existed only in NCF series samples (see Figure 6), and most of the damage onset was correlated to 'micron size' scattering defects from the online monitor during laser damage testing. When continuous filtration was employed, there were no 'micron size' defects observed for the CF series samples. This was the reason for the improvements in LIDT consistency for the CF series samples.

Besides 'micron size' scattering defects, the density of 'sub-micron size' scattering defects in the CF series samples also decreased significantly compared with the NCF series samples. The density in the former was one or two orders of magnitude lower than in the latter. Online monitoring of damage initiation showed that there were a few damage sites $(<10 \%)$ initiated from 'sub-micron size' defects, and most of the damage was not initiated from pre-existing scattering defects (see Figure 9).

The effect of eliminating 'micron size' defects was to lower the damage probability at low fluences, and it did not change the probability of damage at high fluences. However, the curve of damage probability shifted to a higher laser fluence for the $\mathrm{CF}$ samples compared with the NCF

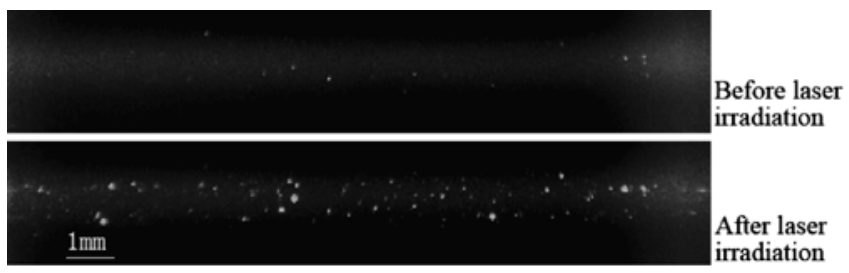

Figure 9. Typical damage initiation in CF series samples.

samples, and 'sub-micron size' scattering defects were not the main cause for damage initiation. That means that there were high-fluence damage precursors other than 'sub-micron size' scattering defects. The pinpoint damage shown in Figure 9(b) showed that the high-fluence damage precursors were high-density distributed absorptive defects. Although there was no direct proof as to the high-fluence damage precursors from the experimental observations, we can get some information about them from the spectra curve. The transmission of the $\mathrm{CF}$ series samples was higher than that of the NCF series samples, with an increasing gap between the two curves on moving from short wavelengths to long wavelengths. The major difference was located in the infrared band, and was greater than $2 \%$. The improvement of transmission may be attributed to decreased scattering losses and absorption losses. The scattering loss was certainly decreased for CF series samples compared with NCF series samples due to the change in scattering defect density shown in Figure 6. However, this was not the main reason for the increased transmission, as scattering is wavelength dependent and higher scattering losses should be expected at shorter wavelengths according to the scattering law. This means that the increasing transmission difference originated from decrease absorption losses, i.e., absorptive defects, giving rise to a shift of the damage probability curve to higher fluences. Therefore, continuous filtration reduced not only the scattering defects but also the absorptive defects, which was the reason for the improved quality of the rapid-grown KDP crystals.

\section{Conclusion}

Rapid growth of KDP crystals with continuous filtration has been demonstrated. The transmittance of the crystals for the infrared band was at least $2 \%$ higher than that of samples grown without continuous filtration, which was attributed to the decrease of absorption. Micron size scattering defects were identified as low-fluence damage precursors, and can be eliminated by continuous filtration. The damage resistance of the crystals was greatly improved due to the elimination of low-fluence damage precursors and the decrease in absorption. 


\section{Acknowledgements}

We are grateful for helpful discussions with Prof. Yuangen Yao of Fujian Institute of Research on the Structure of Matter, and Professors Xinguang Xu and Xun Sun of Shandong University.

\section{References}

1. J. J. De Yoreo, A. K. Burnham, and P. K. Whitman, Int. Mater. Rev. 47, 113 (2002).

2. A. Chernov, N. Zaitseva, and L. Rashkovich, J. Cryst. Growth 102, 793 (1990).

3. N. P. Zaitseva, J. J. DeYoreo, M. R. Dehaven, R. L. Vital, K. E. Montgomery, M. Richardson, and L. J. Atherton, J. Cryst. Growth 180, 255 (1997).

4. N. Zaitseva and L. Carman, Prog. Cryst. Growth Ch. 43, 1 (2001).

5. M. D. Feit and A. M. Rubenchik, Proc. SPIE 5273, 74 (2004).

6. C. H. Chan, Appl. Phys. Lett. 26, 628 (1975).

7. G. Duchateau, Opt. Express 17, 10434 (2009).
8. R. A. Negres, P. DeMange, and S. G. Demos, Opt. Lett. 30, 2766 (2005).

9. C. Marshall, S. Payne, M. Henesian, J. Speth, and H. Powell, J. Opt. Soc. Am. B 11, 774 (1994).

10. G. Duchateau and A. Dyan, Opt. Express 15, 4557 (2007).

11. S. Setzler and K. Stevens, Phys. Rev. B 57, 2643 (1998).

12. C. S. Liu, Q. Zhang, and N. Kioussis, Phys. Rev. B 68, 224107 (2003).

13. C. S. Liu, N. Kioussis, S. G. Demos, and H. B. Radousky, Phys. Rev. Lett. 91, 015505 (2003).

14. K. E. Montgomery and F. P. Milanovich, J. Appl. Phys. 68, 3979 (1990).

15. Y. Nishida, A. Yokotani, T. Sasaki, K. Yoshida, T. Yamanaka, and C. Yamanaka, Appl. Phys. Lett. 52, 420 (1988).

16. B. Dam, P. Bennema, and W. J. P. van Enckevort, J. Cryst. Growth 74, 118 (1986).

17. N. Zaitseva, J. Atherton, R. Rozsa, L. Carman, I. Smolsky, M. Runkel, R. Ryon, and L. James, J. Cryst. Growth 197, 911 (1999).

18. A. Burnham, M. Runkel, M. Feit, A. Rubenchik, R. Floyd, T. Land, W. Siekhaus, and R. Hawley-Fedder, Appl. Opt. 42, 5483 (2003).

19. G. Duchateaua, Proc. SPIE 7504, 75041K (2009). 